\title{
Hybrid Energy Management Technique: A Paradigm Shift to Energy Conservation in Wireless Sensor Networks
}

\author{
Onuekwusi Nnaemeka Chiemezie ${ }^{1}$, Okpara Chinedu Reginald ${ }^{2}$ \\ ${ }^{1,2}$ Department of Electrical and Electronic Engineering, \\ Federal University of Technology, Owerri, \\ Imo State, Nigeria. \\ Ewunonu Toochi. C. ${ }^{3}$ \\ ${ }^{3}$ Department of Cyber Security, \\ Federal University of Technology, Owerri, \\ Imo State, Nigeria.
}

\begin{abstract}
Energy constraint is unarguably a major challenge facing Wireless Sensor Networks (WSN) and their immense benefits. The need to overcome it has made energy management in WSNs a research destination in recent times. In this paper, a review of notable energy management schemes employed in WSNs is undertaken. Findings reveal that although some schemes offer improvements over others, yet no scheme can claim to completely overcome the perennial challenge of energy constraint in WSN. A case is therefore made to encourage research efforts to shift in the direction of the synergistic combination of two or more of the exiting strategies into hybrid techniques for improved energy management.
\end{abstract}

Keywords:- Energy conservation, Wireless sensor networks.

\section{INTRODUCTION}

WSN has become a research hotspot over the years. This is because of its immense capabilities and applications. WSNs have been made viable by the convergence of Micro Electro-Mechanical Systems Technology (MEMS), wireless communications and digital electronics [1]. WSNs have gained varied applications in many areas including transportation [2, 3], security [4], agriculture[5][6] and electric power sector $[7,8,9,10]$. WSN is a network of (possibly low-size and low-complex) devices denoted as nodes that can sense the environment and communicate the information gathered from the monitored field through wireless links, to a sink that can use it locally, or is connected to other networks (e.g., the Internet) through a gateway [11]. Its operational activities mainly comprise of sensing, data processing and communication with the sensor, microprocessor and transceiver respectively responsible for these activities. The Battery is responsible for meeting the power needs of the different functionalities of the node and therefore largely determines the lifetime of a node and by extension a WSN.

Research is consistent in identifying energy constraint as the greatest challenge facing WSNs amongst other challenges like communication range, bandwidth, data rate, data security, network topology, communication protocol, processing speed, frequency of transmission, storage, data processing algorithms, Radio Interference and transmission retries. This paper undertakes a brief review of previously explored WSN energy management schemes, highlighting their merits or otherwise and proposes the need for more attention in the synergistic combination of the desirable attributes of these schemes for improved energy management.

\section{CONTRIBUTIVE FACTORS TO WSN ENERGY CONSUMPTION AND WSN DEFINITION OF LIFETIME}

All operational activities of the sensor node contribute to energy drain and lifetime reduction of WSNs [12]. The communication activity contributes the most significant energy drain in the Sense - Process - Communicate activity of WSNs as identified in $[13,14,15]$. The trend in most research efforts on energy management schemes in WSNs is that relying on the above fact, almost all efforts are concentrated in minimizing communication activity in order to conserve energy and maximise lifetime [16, 17]. However, [18, 19, 20, 21] show that this fact does not hold in a number of practical applications, where the energy consumption of the sensing and processing operations may be comparable to, or even greater than, that of the communication. Incorporating energy awareness into every stage of WSN design and operation, thus empowering the system to make dynamic tradeoffs between energy consumption, system performance, and operational fidelity will be the best approach to maximise energy efficiency and network lifetime [22]. MANAGE

A common critical issue in the many literatures bothering energy dissipation in WSN is the definition of the lifetime of a WSN. The lifetime of a WSN is largely dependent on the definition of the death of the sensor node(s) which in turn greatly depends on the energy depletion in the node(s) [23]. 
The lifetime of an arbitrary WSN ' $\mathrm{X}$ ' with ' $\mathrm{N}$ ' number of nodes where:

$$
\begin{aligned}
& \mathbf{N}=1,2,3, \ldots \mathrm{n} \\
& \mathbf{N}_{(\mathbf{1})}=\text { lifetime of the first node } \\
& \mathbf{N}_{(\mathbf{n})}=\text { lifetime of the last node }
\end{aligned}
$$

can be determined by the following approaches:

- First is when the first node dies denoted by $\left(\mathrm{N}_{(1)}{ }^{-}\right.$ of-N lifetime), used in [24, 25]

- Second is when all nodes die denoted by (n-of-N lifetime), presented in [24, 25, 26, 27] amongst others.

- Thirdly is when a good portion of nodes die denoted by (K-of-N lifetime) and explored in [28]. This approach creates a safe margin between the extremes of the first two approaches. It assumes that ' $\mathrm{X}$ ' is considered alive if $\mathrm{K}$ portions of $\mathrm{N}$ has enough energy to sustain the operation of ' $X$ ' and ' $\mathrm{X}$ ' considered dead if $\mathrm{K}$ portions of $\mathrm{N}$ has little or no energy to sustain the operation of ' $\mathrm{X}$ '.

\section{WSN ENERGY MANAGEMENT SCHEMES}

The invaluable need to maximise the lifetime of WSNs has necessitated the plethora of energy management schemes developed at present. This section presents a review of some of the notable schemes outlining their mechanisms, attributes and limitations.

\section{a. Duty Cycle}

This is perhaps more of a WSN energy management strategy than it is a scheme. It is the fraction of time nodes are active during their lifetime [29]. The duty cycle scheme involves putting the radio transceiver in the low-power sleep mode as soon as there is no more data to send/receive and resuming it as soon as a new data packet becomes ready $[30,31]$. It considerably reduces the energy expended in the transmission of message when the receiver is not ready (over transmitting) and listening to receive possible traffic that is not sent (idle listening). The duty cycle technique involves three operational modes namely: the active mode (when the node is transmitting or receiving, usually power consuming), the idle mode (when the node isn't transmitting or receiving but active enough to sense events, also power consuming but less than that of the active mode) and the low power sleep mode (when the operational activities of the node has been put off to conserve power). The possibility of nodes loosing events when in the low power sleep mode however poses a challenge to the duty cycle technique.

\section{b. Low Energy Adaptive Clustering Hierarchy (LEACH)}

The LEACH is a clustering based protocol that includes the formation of distributed groups. It randomly selects a few nodes as cluster heads and rotates this role to evenly distribute the energy load amongst the nodes of the network [26, 32]. Data collection is centralized and is carried out periodically. The cluster heads compress the data arriving from the nodes in their respective groups, and send summary packets to the base station. This reduces the amount of information transmitted to the base station and thus conserves energy. The challenge in this scheme stems from central role of the cluster heads in the operation of the network and the fact that their energy expenditure is more compared to other nodes. Consequently, the death of any of the cluster heads in most cases will imply the death of the network.

\section{c. Sensor Protocols for Information via Negotiation (SPIN)}

The SPIN protocol operates on the assumption that all nodes in the network are potential base-stations and therefore sends all information from each node to every other node in the network. It adopts this approach on the awareness that nodes in close proximity have similar data and the need to distribute data that other nodes do not posses [33]. Proposed in [34], SPIN conserves energy by its awareness of the current energy level of every node and the adaptation of its protocol on how much energy is remaining. It further conserves energy by sending only relevant data describing the sensors' attributes.

SPIN however runs the challenge of implosion caused by duplicate messages sent to the same node. This situation is energy consuming for both the transmitting node and receiving nodes. Gossip-Based protocols as explored by $[23,35]$ overcomes the challenge of SPIN's implosion by just selecting a random node to send the packet to rather than broadcasting the packet blindly. This however introduces delays in propagation of data through the nodes and random selection of receiving node may introduce reliability issues.

\section{d. Carrier Sense Multiple Access-Based MAC Protocol (CSMA)}

Different versions of the CSMA protocols can be used in WSNs. The CSMA protocol typically involves the transmitting node to listen to the network before sending information [36]. CSMA protocols usually exhibit short delay and good performance under low traffic load conditions. However, as traffic increases, collision probability also increases and CSMA protocols efficiency decreases with a consequent adverse effect on the energy resource of the network. Although the proposition of [37] to mitigate this effect by the application of constant listen periods and random delays is viable, it does not completely eliminate the challenge.

\section{e. Energy and Rate-Based MAC Protocol (ER- $M A C)$}

The ER-MAC presented in [38], employs the TDMA technique to avoid energy waste. It conserves energy by using the concept of periodic listen and sleep. Each node is assigned two TDMA slots for transmission and knows the transmission slots of its neighbours. Nodes periodically share information about their power levels and determine whether to use one or two slots for transmission [39]. ERMAC completely eliminates energy waste due to packet loss from collisions because two nodes do not transmit in the same time slot. It is however saddled with the challenge of packet losses from interference and loss of signal strength. 


\section{f. Energy Harvesting}

The potentials of harvesting ambient energy sources to prolong the lifetime of WSNs have also been explored. The energy harvested from a variety of ambient sources (heat, solar, wind, vibration, radio frequency radiation) provides possible means to recharge the nodes batteries or eliminate them entirely [40]. Table 1 outlines the power densities of some energy harvesting technologies.

Table 1: Ambient Power Sources for WSN [41]

\begin{tabular}{|l|l|}
\hline Harvesting Technology & Power Density \\
\hline Solar Cells & $15 \mathrm{~mW} / \mathrm{cm}^{2}$ \\
\hline Piezoelectric & $330 \mu \mathrm{W} / \mathrm{cm}^{3}$ \\
\hline Vibration & $116 \mu \mathrm{W} / \mathrm{cm}^{3}$ \\
\hline Thermoelectric $10^{\circ} \mathrm{C}$ & $40 \mu \mathrm{W} / \mathrm{cm}^{3}$ \\
\hline Acoustic noise $(100 \mathrm{~dB})$ & $960 \mathrm{nW} / \mathrm{cm}^{3}$ \\
\hline
\end{tabular}

Solar cells which have the highest power density amongst the compared technologies offer the most attractive way for energy harvesting in WSN [42] followed by piezoelectric and vibration sources. Each form of energy is harvested by a different class of generator that performs conversion to electricity.

The challenge in the use of energy harvesting in WSN includes that for all sources, the energy is available only under particular environmental conditions like the presence of sunlight, vibration, high temperature and surrounding noise. Also, efficiency may decrease due to the direct connection between harvesting components and energy storage devices. The decrease in efficiency can be accounted for by the efficiency of converting one form of energy to another, efficiency of transferring from the source to the supply, efficiency of buffering after the energy has been harvested and finally, consumption efficiency. These reasons inform why wireless sensor nodes usually keep energy in storage elements. To address these challenges, combination of an energy storage system and scavenging technology would be a reasonable strategy for optimal advantage.

\section{g. Multiple Battery Levels}

Studies carried out in [43] observed that regardless of the routing strategy, MAC layer, physical layer considerations etc the sensor nodes closer to the gateway have to forward more packets than the ones at the periphery of the network. On analysis of their observation, they assumed that the increase in workload will result to an increase in power consumption causing the nodes close to the gateway to die first, leading to a premature loss of connectivity in the sensor network. They further observed that depending on network size and the actual power consumed for transmission and reception, that there exists the possibility that a large percentage of nodes away from the gateway will have almost full batteries and yet be incapable of reaching the base station as a result of loss in connectivity with those near the gateway.
To alleviate this undesirable effect, they demonstrate the use of multiple levels of batteries, placed concentrically around the base station. They show through simulation that given a total energy budget for the sensor network, the lifetime of the network can be significantly improved by the adoption of the multiple battery level approach. Against the merit of the multiple battery level approach, the scheme is however limited to the structure-based WSN architecture. It failed to account for the unstructured architecture and applications that may require all nodes to originate data, forward same to other node(s) and also forward those received from other node(s). It also has limitation in unstructured WSN architecture scenarios that may require the random deployment of nodes.

\section{h. Artificial Intelligence (AI) Techniques}

The use of AI techniques is yet another explored strategy for effective management of resources (energy inclusive) in event monitoring applications like WSNs. AI mimics human perception, learning and reasoning to solve complex problems. Studies in [44] outline some of the AI techniques to include: case-based reasoning, rule-based systems, artificial neural networks, fuzzy models, genetic algorithms and swarm intelligence.

The case-based reasoning technique explored in [45, 46, 47] recognises that problems are easier to solve by repeated attempts resulting in accrued learning. It solves a problem by recalling similar past problems assumed to have similar solutions. It relies on numerous past cases to adapt its solutions or methods to a new problem of diagnosis, prediction, control and planning. The RBS is yet another AI technique that can be applied in resource management in WSNs. It generates its solutions from established rules. It involves no learning, cannot automatically add or modify rules and as such has its application quite limited. RBS are well suited to solve problems where comprehensive expert knowledge is available to articulate decisions confidently [48]. The rules have the condition ('if') part and action ('then') part which are fed to an inference engine, which has a working memory of information about the problem, a pattern matcher and a rule applier.

Of the existing AI techniques, ANN has in recent times been significantly explored in energy management of WSNs. ANN is an AI technique that imitates the way the human brain processes information. It acquires knowledge through learning and its knowledge is stored within inter neuron connection strengths known as synaptic weights [49]. It consists of many processing units (neurons or nodes) working in unison with the highly interconnected weighted links called synapses. Studies in [50] attributes the interest in the exploitation of ANN in energy management in WSN as what they describe as the analogy between the two. It is their viewpoint that ANNs exhibit exactly the same architecture as WSNs, with the ANN neurons corresponding to WSN's sensor nodes and the ANN connections corresponding to radio links. In this light, WSN can be viewed as an ANN and an ANN algorithm can be run on each sensor node to decide on the 
output action. Some of the algorithms developed within the classical artificial neural networks, can be easily adopted to WSN platforms and meet requirements like: energy management, simple-parallel distributed computation, distributed storage, data robustness and auto-classification of sensor readings, fault tolerance and low computation [44].

\section{i. Hybrid Approach}

From reviewed literatures on energy conservation and management schemes, no particular scheme has proved to be most effective. A particular scheme preferred in one application scenario may appear undesirable in other applications. This has necessitated the insight on the synergistic combination of two or more schemes for improved energy efficiency and to achieve wider areas of applications [51 - 52].

It is noteworthy that the hybrid approach is not an energy management technique on its own, but rather creates a platform for the cooperative combination of some of the reviewed energy management techniques. For instance a combination of the Multiple Battery Level scheme can be explored to overcome the challenge experienced in the LEACH and other hierarchical WSN energy management schemes. Also the predictive attribute of the ANN can also be exploited to overcome the possibility of event miss experienced by the duty cycle technique. The synergistic combination opportunity of the hybrid approach can indeed be leveraged for improved energy management.

\section{CONCLUSION}

The development of an efficient energy management strategy occupies a central position in the operation and lifetime of WSN. The observation that the common existing strategies have limitations amplifies the need for their improvement. For improved efficiency of these schemes and the maximization of the lifetime of WSNs, the hybrid approach is indeed a necessary strategy to explore.

\section{REFERENCES}

1 I. F. Akyildiz, W. Su, Y. Sankarasubramaniam, and E. Cayirci. "Wireless Sensor Networks: A Survey". Computer Networks, Vol 38 , pp. $393-422,2002$.

2 U.S. Department of Transportation. "Intelligent Transportation Systems Applications Overview" Research and Innovative Technology Administration, (RITA). Internet: www.itsoverview.its.dot.gov/, 2009 [Accessed July 1, 2019].

3 Intelligent Transportation Systems Society (ITS Canada). "About Intelligent Transportation Systems (ITS)", Internet: www.itscanada.ca/english/aboutits.htm, 2009. [Accessed July, 1, 2019].

4 D. Okereafor, U. Diala, N. Onuekwusi, L. Uzoechi and G. Chukwudebe. "Improving Security and Emergency Response Through the Use of Unmanned Vehicles". IEEE Nigeria Section 2nd International Conference on Emerging \& Sustainable Technologies for Power \& ICT in a Developing Society (NIGERCON), Owerri, Nigeria, November $14-16,2013$.

5 T. Wark, P. Corke, P. Sikka, L. Klingbeil, Y. Guo, C. Crossman, P. Valencia, D. Swain and G. Hurley. "Transforming Agriculture through Pervasive Wireless Networks". Pervasive Computing, pp 50-57, 2007.

6 R. Evans and J. Bergman, "Relationships between Cropping Sequences and Irrigation Frequency under Self-Propelled Irrigation Systems in the Northern Great Plains". USDA Annual Report, 2003
7 V. Weber, "Smart Sensor Networks: Technologies and Applications for Green Growth", OECD Conference on ICTs, the Environment and Climate Change, Helsingør, Denmark. May 2009.

8 R. Atkinson, and D. Castro, "Digital Quality of Life Understanding the Personal \& Social Benefits of the Information Technology Revolution", The Information Technology and Innovation Foundation, Washington DC. October 2008

9 H. Siderius and A. Dijkstra. "Smart Metering for Households: Costs and Benefits for the Netherlands", Internet: http://mail.mtprog.com/CD_Layout/Day_2_22.06.06/09001045/I D57 Siderius final.pdf, 2006 [Accessed July 1, 2019]

10 N. Onuekwusi, I. Achumba, N. Chukwuchekwa, G. Chukwudebe and G. Ononiwu. "Leveraging Wireless Sensor Networks for Improved Infrastructure Management in the Energy Sector' Nigerian Society of Engineers Annual National Conference, Akure, Nigeria. November 16 - 20, 2015.

11 S. La Malfa, "Wireless Sensor Networks", Jan, 2010. Internet: http://www.dees.unict.it/users/bando/files/wsn.pdf [Accessed July 7, 2019].

12 P. Kaur and V. Kumar, "A Survey of Energy Optimization Techniques in Wireless Sensor Networks", International Journal of Advanced Research in Computer and Communication Engineering, Vol. 4, Issue 5. May 2015.

13 N. Onuekwusi, M.Ndinechi, G. Ononiwu, O. Nosiri. An Energy Balanced Routing Hole and Network Partitioning Mitigation Model for Homogeneous Hierarchical Wireless Sensor Networks. International Journal of Interdisciplinary Telecommunications and Networking. Vol. 12, Issue 1. pp 28-42. 2020.

14 Q. Wang, M. Hempstead and W. Yang, "A Realistic Power Consumption Model for Wireless Sensor Network Devices". Division of Engineering and Applied Sciences Harvard University. $2005 . \quad$ Internet: http://citeseerx.ist.psu.edu/viewdoc/download?doi=10.1.1.136.10 84\&rep=rep1\&type=pd Accessed August 3, 2019.

15 Y. Kubo, K. Yanagihara and M. Nozak "Electric Power Conservation Technology for Wireless Sensor Networks" Ok Technical Review Special Edition on the Environment: Aiming for Low Carbon Society. Vol.76, Issue 214, No.1. April 2009.

16 G. Anastasi, M. Conti, M. Di Francesco and A. Passarella, "Energy conservation in wireless sensor networks: A survey". Ad Hoc Network, Vol 7, pp 537 - 568. 2009.

17 M. A. Razzaque, C. Bleakley and S. Dobson, "Compression in Wireless Sensor Networks: A Survey and Comparative Evaluation". ACM Trans. Sens. Netw. Vol. 10, Issue 5. 2013.

18 C. Alippi, G. Anastasi, M. Di Francesco and M. Roveri, "Energy Management in Wireless Sensor Networks with Energy-Hungry Sensors. IEEE Instrum. Meas. Mag. Vol 12, pp 16 - 23, 2009.

19 P. Dutta, M. Grimmer, A. Arora, S. Bibyk and D. Culler, "Design of a Wireless Sensor Network Platform for Detecting Rare, Random, and Ephemeral Events". Proceedings of the 4th International Symposium on Information Processing in Sensor Networks. Los Angeles, CA, USA. 25 - 27 April 2005.

20 M. A. Razzaque and S. Dobson, "Energy-Efficient Sensing in Wireless Sensor Networks Using Compressed Sensing”, 2014 Internet: www.mdpi.com/journal/sensors. [Accessed August 3, 2019].

21 C. Buratti, A. Conti, D. Dardari and R. Verdone, "An Overview on Wireless Sensor Networks Technology and Evolution". 2009. Internet: www.mdpi.com/journal/sensors. [Accessed August 3, 2019].

22 V. Raghunathan, C. Schurgers, S. Park, M. Srivastava and B. Shaw, "Energy-Aware Wireless MicroSensor Networks". IEEE Signal Process. Vol 19, pp 40 - 50. May 2002.

23 M. G. C. Torres, "Energy Consumption In Wireless Senso Networks Using GSP” Unpublished M.Sc Thesis Submitted To The Graduate Faculty Of The School Of Information Sciences, University Of Pittsburgh, USA. April, 2006.

24 M. Bhardwaj and A .P. Chandrakasan, "Bounding the Lifetime of Sensor Networks Via Optimal Role Assignments". IEEE INFOCOM. 2002.

25 J. Chang and L. Tassiulas, "Maximum Lifetime Routing in Wireless Sensor Networks", IEEE INFOCOM, Tel Aviv, Israel 2000 
26 W. Heinzelman, A. Chandrakasan and H. Balakrishnan, "Energy Efficient Communication Protocol for Wireless Microsensor Networks". Proceedings of the 33rd Hawaii International Conference on System Sciences (HICSS '00). January 2000.

27 X. Hou, D. Tipper, D. Yupho and J. Kabara, "GSP: Gossip-based Sleep Protocol for Energy Efficient Routing in Wireless Sensor Networks", The 16th International Conference on Wireless Communications, Calgary, Alberta, Canada. 2004.

28 A. Ephremides, "Energy concerns in Wireless Networks", IEEE Wireless Communications. Aug 2002.

29 V. K. Sachan, S. A. Imam and M. T. Beg "Energy-efficient Communication Methods in Wireless Sensor Networks: A Critical Review", International Journal of Computer Applications. Vol. 39, Issue 17. February 2012.

30 J. Saraswat and P. P. Bhattacharya, "Effect of Duty Cycle on Energy Consumption in Wireless Sensor Networks", International Journal of Computer Networks \& Communications (IJCNC) Vol.5, Issue1. January 2013.

31 A. Moschitta and I. Neri, "Power Consumption Assessment in Wireless Sensor Networks". Chapter 9 of ICT - Energy Concepts Towards Zero - Power Information and Communication Technology. 2014. Internet: http://cdn.intechopen.com/pdfswm/45946.pdf [Accessed August 10, 2019].

32 A. Chaibrasou and A. Mouhsen, "General Approach for the Conservation of Energy in Wireless Sensor Networks", International Journal of Computer Science Issues (IJCSI), Vol. 10, Issue 2, No 2. March 2013

33 J. N. Al-Karaki and A. E. Kamal, "Routing Techniques in Wireless Sensor Networks: A Survey", Wireless Communications, IEEE. Vol.11, Issue 6, pp. 6 - 28. Dec. 2004

34 J. Kulik, W. R. Heinzelman, and H. Balakrishnan, "NegotiationBased Protocols for Disseminating Information in Wireless Sensor Networks," Wireless Networks. Vol. 8, pp. 169 - 185. 2002.

35 Z. J. Haas, J. Y. Halpern and L. Li, "Gossip-Based Ad Hoc Routing," Proceedings of $21^{\text {st }}$ Annual Joint Conference of the IEEEComputer and Communications Societies. NewYork, USA. June $23-27,2002$.

36 A. Tanenbaum, "Computer Networks". 4th Edition. Prentice Hall, Upper Saddle River, New Jersey. 2003.

37 A. Woo, and D. Culler, "A Transmission Control Scheme for Media Access in Sensor Networks," Proceedings of ACM MobiCom. Rome, Italy. July 2001.

38 R. Kannan, R. Kalidindi, S. S. Iyengar and V. Kumar, "Energy and Rate Based MAC Protocol for Wireless Sensor Networks". Special Section on Sensor Network Technology and Sensor Data Management, Vol. 32 , Issue 4, pp. 60-65. December 2003.

39 S. Sendra, J. Lloret, M. García and J. F. Toledo, "Power Saving and Energy Optimization Techniques for Wireless Sensor Networks", Journal of Communications, Vol. 6, Issue. 6. September 2011.

40 D. Zhou, N. Kong, D. S. Ha and D. J. Inman, "A Self-Powered Wireless Sensor Node for Structural Health Monitoring",
Proceedings of SPIE Health Monitoring of Structural and Biological Systems. San Diego, CA, USA. 2010.

41 S. Roundy, P. Wright, and J. Rabaey, "A study of Low Level Vibrations as a Power Source for Wireless Sensor Nodes". Computer Communications. Vol. 26, Issue. 11. Pp 1131 - 1144. July 2003.

42 A. Somov, "Power Management and Power Consumption Optimization Techniques in Wireless Sensor Networks", unpublished $\mathrm{Ph} . \mathrm{D}$ dissertation submitted to the International Doctorate School in Information and Communication Technologies" University of Trento, Italy. 2009.

43 M. Sichitiu, R. Dutta. "Benefits of Multiple Battery Levels for the Lifetime of Large Wireless Sensor Networks". In: Boutaba R., Almeroth K., Puigjaner R., Shen S., Black J.P. (eds), Networking Technologies, Services, and Protocols; Performance of Computer and Communication Networks; Mobile and Wireless Communications Systems. Springer, Berlin, Germany.2005

44 S. H. Chen, A. J. Jakeman and J. P. Norton, "Artificial Intelligence Techniques: An Introduction to their use for Modelling Environmental Systems", Science direct-Mathematics and Computers in Simulation. Vol. 78, Issue 3,pp 379 - 400. 2008

45 E. Kalapanidas and N. Avouris, "Short-term Air Quality Prediction using a Case-based Classifier". Environmental Modelling and Software, Vol.16, Issue. 3. pp 263 - 272. 2001

46 D. S. Kaster, C. B. Medeiros and H. V. Rocha, "Supporting Modelling and Problem Solving from Precedent Experiences: The Role of Workflows and Case-based Reasoning", Environmental Modelling and Software, Vol.20, Issue. 6. Pp 689-704. 2005

47 H. Nunez, M. Sanchez-Marre, U. Cortes, J. Comas, M. Martinez, I. Rodriguez-Roda and M. Poch, "A Comparative Study on the use of Similarity Measures in Case-based Reasoning to Improve the Classification of Environmental System Situation". Environmental Modelling and Software, Vol. 19, Issue. 9. pp 809 $-819.2004$

48 V. Dhar and R. Stein, "Intelligent Decision Support Methods", The Science of Knowledge Work. Prentice Hall, New Jersey, 1997.

49 A. Nanda, B. Dinda, R. Rath and S. Pradhan, "Wireless Sensor Network For Super Cyclone Prediction Using Artificial Neura Network", Asian Journal Of Computer Science And Information Technology, Vol. 1, Issue 2. pp 30 - 33. 2011.

50 F. Oldewurtel and P. Mahonen, "Neural Wireless Sensor Networks". IEEE International Conference on Systems and Networks Communications (ICSNC '06). Tahiti. October 2006.

51 P. Leela and K. Yogitha, "Hybrid Approach for Energy Optimization in Wireless Sensor Networks", International Journal of Innovative Research in Science, Engineering and Technology. Vol. 3, Issue 3. 2014.

52 S. Thangavelu and S. Shanmugavel, "Hybrid Approach for Energy Optimization in Wireless Sensor Networks using ABC and Firefly Algorithms". International Review on Computers and Software, Vol. 8, Issue 10. pp 2335 - 2341. 2013. 